\title{
Outcomes after stereotactic radiosurgery of brain metastases in patients with malignant melanoma and validation of the melanoma molGPA
}

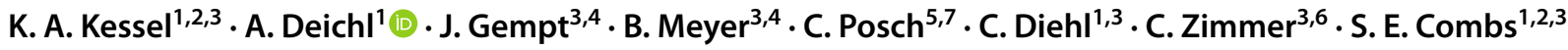

Received: 12 January 2021 / Accepted: 22 March 2021 / Published online: 15 May 2021

(C) The Author(s) 2021

\begin{abstract}
Introduction Malignant melanoma is the third most common primary in the diagnosis of brain metastases. Stereotactic radiosurgery (SRS) is a well-established treatment option in limited brain disease. We analyzed outcomes of SRS with a particular focus on the graded prognostic assessment (GPA, melanoma molGPA), prognostic factors, and toxicity.

Methods We evaluated 173 brain metastases in 83 patients with malignant melanoma. All were treated with SRS median dose of 20 Gy prescribed to the 80 or 100\% isodose line between 2002 and 2019. All patients were followed-up regularly, including contrast-enhanced brain imaging as well as clinical examination, initially 6 weeks after treatment, then in quarterly follow-up.

Results The median age was 61 years (range 27-80); 36 female and 47 male patients were treated. After a median follow-up of 5.7 months, median OS (overall survival) was 9.7 months 95\%-KI 4.7-14.7). LC (local control) at 6 months, 12, 24 months was $89 \%, 86 \%$, and $72 \%$, respectively (median was not reached). Median DBC (distant brain control) was 8.2 months (95\%KI 4.7-11.7). For OS, a KPS $\geq 80 \%$, a positive BRAF mutation status, a small PTV (planning target volume), the absence of extracranial metastases, as well as a GPA and melanoma molGPA $>2$ were prognostic factors. In the MVA, a small PTV and a melanoma molGPA $>2$ remained significant.

Conclusion The present survival outcomes support the use of the disease-specific melanoma molGPA as reliable prognostic score. Favorable outcomes for SRS compared to other studies were observed. In the treatment of brain metastases of malignant melanoma patients, a multidisciplinary approach consisting of surgery, SRS, chemotherapy, and immunotherapy should be considered.
\end{abstract}

Keywords Brain metastases $\cdot$ Melanoma $\cdot$ SRS $\cdot$ Radiosurgery $\cdot$ GPA $\cdot$ Prognostic factors

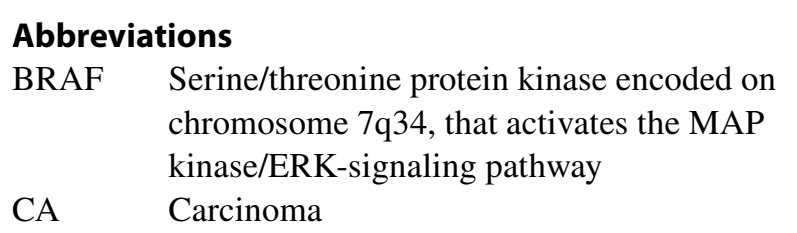

K. A. Kessel and A. Deichl contributed equally.

A. Deichl

andreadeichl@gmail.com

1 Department of Radiation Oncology, Klinikum rechts der Isar, Technical University Munich (TUM), Ismaninger Straße 22, 81675 Munich, Germany

2 Institute of Radiation Medicine (IRM), Helmholtz Zentrum München, Neuherberg, Germany

3 Deutsches Konsortium für Translationale Krebsforschung (DKTK), DKTK Partner Site Munich, Munich, Germany
CT Contrast-enhanced computed tomography

CTCAE Common toxicity criteria

CTLA-4 Cytotoxic T-lymphocyte-associated protein 4

$\mathrm{D}_{\max } \quad$ Maximum dose to the PTV

DBC Distant brain control

dsGPA Disease-specific GPA

4 Department of Neurosurgery, Technical University of Munich (TUM), Munich, Germany

5 Department of Dermatology and Allergy, Technical University of Munich (TUM), Munich, Germany

6 Department of Neuroradiology, Technical University of Munich (TUM), Munich, Germany

7 Faculty of Medicine, Sigmund Freud University, Vienna, Austria 


$\begin{array}{ll}\text { EFS } & \text { Event-free survival } \\ \text { FSRT } & \text { Fractionated stereotactic radiotherapy } \\ \text { GKR } & \text { Gamma knife radiosurgery } \\ \text { GPA } & \text { Graded prognostic assessment } \\ \text { IGRT } & \text { Image-guided radiotherapy } \\ \text { IQR } & \text { Interquartile range } \\ \text { KPS } & \text { Karnofsky performance score } \\ \text { LC } & \text { Local control } \\ \text { MM } & \text { Malignant melanoma } \\ \text { MVA } & \text { Multivariate analysis } \\ \text { NR } & \text { Not reported } \\ \text { OS } & \text { Overall survival } \\ \text { PD-1 } & \text { Programmed cell death protein 1 } \\ \text { PTV } & \text { Planning target volume } \\ \text { RCC } & \text { Renal cell cancer } \\ \text { RPA } & \text { Recursive partitioning analysis } \\ \text { RT } & \text { Radiotherapy } \\ \text { RTOG } & \text { Radiation therapy oncology group } \\ \text { SRS } & \text { Stereotactic radiosurgery } \\ \text { UVA } & \text { Univariate analysis } \\ \text { WBRT } & \text { Whole-brain radiotherapy }\end{array}$

\section{Introduction}

Patients with solid malignancies often develop brain metastasis at some point during their disease. Metastases are the most common brain neoplasm and may spread from any primary disease [1]. In up to $40 \%$ of patients with solid primary tumors outside the central nervous system, brain metastases are diagnosed, and the incidence is continuously increasing [2]. Only $10 \%$ of patients survive more than 1 year [3].

The treatment of brain metastases relies on local strategies, including surgery, stereotactic radiosurgery (SRS), as well as on whole-brain radiation (WBRT). First-line treatment decisions in patients with newly diagnosed brain metastases mainly rely on the primary tumor, number and size of brain metastases and Karnofsky performance score (KPS), which are considered in the newly established graded prognostic assessment (GPA). Primarily the GPA was developed as a new score in 2008 and based on an analysis of 1960 patients whose data were extracted from the Radiation Therapy Oncology Group (RTOG) database. The GPA includes four criteria: age, KPS, number of brain metastases, and the presence/absence of extracranial metastases [4]. Based on a retrospective study of $>4000$ patients with brain metastases, Sperduto et al. determined various prognostic factors depending on the histology of the primary and modified the GPA to so-called disease-specific GPA (dsGPA) scores. For patients with malignant melanoma, the updated prognostic score is a further development of the GPA-the so-called melanoma molGPA-is a more complex assessment, which also includes the BRAF mutation status [5-7].
SRS has become increasingly important, and a wellaccepted treatment method for brain metastases $[8,9]$. The technique relies on multiple beams of radiation intersecting at a target precisely located within three dimensions [8]. For brain metastases, SRS can be delivered via linear accelerator or gamma knife [10] and is now used in a variety of clinical scenarios for patients with brain metastases, including as a boost WBRT therapy, as a definitive treatment alone for patients with a limited number of brain metastases, and in the pre- or postoperative setting [10].

Malignant melanoma is the third most common primary in the diagnosis of brain metastases after lung carcinoma, and breast cancer [11]. Brain metastases occur in up to $50 \%$ of patients with metastatic melanoma [12]. The chances of being cured are good in the early stages of the disease, but the median overall survival (OS) of patients with untreated brain metastases is less than 3 months [13].

Up until 2011, no systemic therapy had convincingly been shown to improve the survival of patients with metastatic melanoma [14]. Since the last decade, two different treatment concepts are available for targeted therapy, $\mathrm{BRAF} \pm \mathrm{MEK}$ inhibition $[15,16]$, and immune checkpoint blockade [17, 18], which confirmed a survival benefit for advanced melanoma patients [14]. They represent two very distinctive classes of agents: small molecule inhibitors of the MAP kinase signaling pathway and monoclonal antibodies against immune checkpoint molecules [14]. In $40-60 \%$ of malignant melanomas, there is a mutation in the BRAF gene that leads to the activation of a signal transduction pathway relevant for tumor development [19]. Since the drugs impressively control extracranial metastasis, the therapy of brain metastasis is of new relevance [20]. Additionally, the availability of further novel immunotherapeutic drugs will be upcoming, which will continuously improve outcomes. Therefore, the need for non-invasive, highly effective treatments for brain metastases becomes more imminent.

Malignant melanoma brain metastases have traditionally been considered 'radioresistant' to conventional fractionated external beam radiotherapy (FSRT) and WBRT [21]. Hence, in the past decade, SRS has become a well-established treatment modality with high efficacy as it delivers a high dose to the lesion [21]. In selected patients with one to four metastases measuring less than 3-4 cm, SRS yields an excellent local and distant brain control (LC, DBC), more than $85 \%$, and median survival of 5-11 months [22].

A multidisciplinary approach consisting of surgery, radiotherapy, chemotherapy, and immunotherapy has proved beneficial in advanced stages of metastasis of malignant melanoma [23].

In the present study, we analyze outcomes (OS, LC, DBC) after SRS of brain metastases in patients with malignant melanoma. We further aim to investigate the graded 
prognostic assessment (GPA, melanoma molGPA), prognostic factors, and toxicity.

\section{Patients and methods}

\section{Patients}

We retrospectively evaluated 173 brain metastases and 83 patients with malignant melanomas. All were treated with SRS at the Department of Radiation Oncology, Technical University of Munich, between 2002 and 2019. Table 1 displays patient characteristics. The ethics committee of the Medical Faculty of the Technical University Munich (TUM) approved this study and waived patient informed consent: 257/16 S (01.06.2016).

\section{Treatment}

SRS treatment was performed using a thermoplastic mask system (Brainlab, Germany) and daily image-guidance (IGRT) by robotic ExacTrac positioning (Brainlab, Germany). Within the stereotactic localization system, contrastenhanced computed tomography (CT) and T1-weighted magnetic resonance images (MRI) were acquired with a slice thickness of $3 \mathrm{~mm}$.

We fused the images and defined the planning target volume (PTV) as residual macroscopic tumor tissue with a margin of 1-2 $\mathrm{mm}$ for residual uncertainties.

We applied a median total dose of 20 Gy (range: $18-20 \mathrm{~Gy})$ prescribed to the 80 or $100 \%$ isodose line. A Clinac Trilogy linear accelerator equipped with a $120 \mathrm{HD}$ multi-leaf collimator (Varian Medical Systems, Palo Alto, CA, USA) and $6 \mathrm{MV}$ photons was used for irradiation. Eleven patients (13.3\%) received a simultaneously immune therapy or BRAF inhibitor. Parallel chemotherapy was administered in two patients (2.4\%). Before SRS, 17 (17/83, $20.5 \%$ ) patients were already pre-treated with WBRT within a median time interval of 98 days (range 15 days-3.2 years) prior to SRS and a median total dose of $30 \mathrm{~Gy}$ (range: 30-45 Gy).

\section{Follow-up}

All patients were followed-up regularly, including contrastenhanced brain MRI (T1-weighted) and a clinical examination. The first follow-up was performed 6 weeks after the end of treatment, then in a quarterly follow-up with physical examination, blood chemistry and magnetic resonance imaging for local control. After 2 years of recurrence-free survival, the intervals were prolonged individually. In the case of local or distant intracranial failure, salvage therapy was performed after interdisciplinary discussion (neurosurgical
Table 1 Patient characteristic (patients $n=83$, lesions $n=173$ )

\begin{tabular}{|c|c|c|}
\hline Description & Value & $\%$ \\
\hline \multicolumn{3}{|l|}{ Age at RT [years] } \\
\hline Median & 61 & \\
\hline Range & $27-80$ & \\
\hline \multicolumn{3}{|l|}{ Gender } \\
\hline M & 47 & 56.6 \\
\hline $\mathrm{F}$ & 36 & 43.4 \\
\hline \multicolumn{3}{|l|}{ BRAF mutation } \\
\hline Yes & 19 & 22.9 \\
\hline No & 27 & 32.5 \\
\hline Unknown & 37 & 44.6 \\
\hline \multicolumn{3}{|l|}{ KPS at RT [\%] } \\
\hline 100 & 17 & 9.8 \\
\hline 90 & 77 & 44.5 \\
\hline 80 & 52 & 30.1 \\
\hline 70 & 23 & 13.4 \\
\hline 60 & 3 & 1.7 \\
\hline 50 & 1 & 0.6 \\
\hline \multicolumn{3}{|c|}{ Simultaneously immune therapy } \\
\hline Yes & 11 & 13.3 \\
\hline No & 72 & 86.7 \\
\hline \multicolumn{3}{|c|}{ Immune and BRAF therapy agent } \\
\hline Ipilimumab & 1 & 9.1 \\
\hline Nivolumab & 3 & 27.3 \\
\hline Nivolumab \& Ipilimumab & 3 & 27.3 \\
\hline Pembrolizumab & 2 & 18.2 \\
\hline Vemurafenib & 2 & 18.2 \\
\hline \multicolumn{3}{|l|}{ Parallel chemotherapy } \\
\hline Yes & 2 & 2.4 \\
\hline No & 81 & 97.6 \\
\hline \multicolumn{3}{|l|}{ Chemotherapy agent } \\
\hline Temozolomid & 1 & 50.0 \\
\hline Fotemustin & 1 & 50.0 \\
\hline \multicolumn{3}{|l|}{ Prior WBRT } \\
\hline Yes & 17 & 20.5 \\
\hline Dose [Gy] & $30 / 10$ & 70.6 \\
\hline Dose [Gy] & $40 / 20$ & 29.4 \\
\hline No & 66 & 79.5 \\
\hline \multicolumn{3}{|l|}{ Number of metastases at RT } \\
\hline 1 & 29 & 16.8 \\
\hline $2-3$ & 78 & 45.1 \\
\hline$\geq 4$ & 66 & 38.2 \\
\hline \multicolumn{3}{|l|}{ Extracranial metastases } \\
\hline Yes & 66 & 79.5 \\
\hline No & 17 & 20.5 \\
\hline \multicolumn{3}{|l|}{ GPA } \\
\hline $0-1.0$ & 43 & 24.9 \\
\hline $1.5-2.0$ & 99 & 57.2 \\
\hline $2.5-3.0$ & 27 & 15.6 \\
\hline $3.5-4.0$ & 4 & 2.3 \\
\hline
\end{tabular}


Table 1 (continued)

\begin{tabular}{llr}
\hline Description & Value & $\%$ \\
\hline Melanoma molGPA & & \\
$0-1.0$ & 26 & 15.0 \\
$1.5-2.0$ & 101 & 58.4 \\
$2.5-3.0$ & 36 & 20.8 \\
$3.5-4.0$ & 10 & 5.8
\end{tabular}

Time from primary diagnosis to brain metastasis [months]

$\begin{array}{ll}\text { Median } & 36.3 \\ \text { IQR } & 81.8 \\ \text { PTV [ml] } & \\ \text { Median } & 1.0 \\ \text { IQR } & 2.9 \\ D_{\max }(\mathrm{PTV}) \text { [Gy] } & \\ \text { Median } & 24.9 \\ \text { IQR } & 1.7\end{array}$

- Number according to patients, KPS Karnofsky performance score, $B R A F$ Serine/threonine protein kinase, encoded on chromosome $7 \mathrm{q} 34$, that activates the MAP kinase/ERK-signaling pathway, GPA Graded prognostic assessment according to [24, 25], Melanoma mol$G P A$ Disease specific GPA for melanoma patients according to [26], $W B R T$ Whole brain radiotherapy, PTV Planning target volume, IQR Interquartile range

intervention with/without adjuvant radiation therapy performed as WBRT or radiosurgery). Leptomeningeal failure and radiation-induced brain necrosis were confirmed by an interdisciplinary board and determined either after surgery and histopathological examination or on MRI.

Neurologic toxicities were classified as acute if they occurred during treatment or up to the first 6 weeks after the end of SRS. If they occurred later, they were considered as late toxicities.

\section{Statistics}

Primary endpoints were OS, LC (defined as the time to first local failure (LF), which is determined by new contrast enhancement of the previously irradiated metastasis on MRI confirmed by experienced radiologists), and DBC (defined as the time to first loco-regional, intra-cranial failure (LRF), hence, the growth of new or not-treated brain metastases). Secondary objectives included the assessment of GPA (initial GPA and melanoma molGPA, Table 3) and the analysis of prognostic factors as well as neurologic toxicity.

Continuous data were expressed as median and IQR (interquartile range), categorical data as frequency counts and percentages. For calculation of outcomes, we used all treatment cases for LC $(n=173)$ and the first treatment for OS and DBC $(n=83)$. We calculated OS from the last treatment day until the last follow-up visit or death; LC/DBC was calculated from the last treatment day until the date of local/
Table 2 Median and life table for OS, LC, and DBC

\begin{tabular}{lcllll}
\hline & \multirow{2}{*}{$\begin{array}{l}\text { Median } \\
{[\text { months }]}\end{array}$} & \multicolumn{4}{l}{ The proportion of patients surviving after } \\
\cline { 3 - 6 } & & 6 months & 12 months & 18 months & 24 months \\
\hline OS & 9.7 & $67 \%$ & $46 \%$ & $35 \%$ & $32 \%$ \\
LC & - & $89 \%$ & $86 \%$ & $82 \%$ & $72 \%$ \\
DBC & 8.2 & $56 \%$ & $44 \%$ & $34 \%$ & $26 \%$ \\
\hline
\end{tabular}

Table 3 Calculation scheme for the GPA and melanoma molGPA

\begin{tabular}{|c|c|c|c|}
\hline & Prognostic factor & & Score value \\
\hline \multirow[t]{11}{*}{ GPA } & \multirow[t]{3}{*}{ Age [years] } & $<50$ & 1 \\
\hline & & $\geq 50<60$ & 0.5 \\
\hline & & $\geq 60$ & 0 \\
\hline & \multirow[t]{3}{*}{ KPS [\%] } & $100-90$ & 1 \\
\hline & & $80-70$ & 0.5 \\
\hline & & $\leq 60$ & 0 \\
\hline & \multirow[t]{2}{*}{ Extracranial metastases } & No & 1 \\
\hline & & Yes & 0 \\
\hline & \multirow{3}{*}{$\begin{array}{l}\text { Number of brain } \\
\text { metastases }\end{array}$} & 1 & 1 \\
\hline & & $2-3$ & 0.5 \\
\hline & & $\geq 4$ & 0 \\
\hline \multirow{12}{*}{$\begin{array}{c}\text { Mela- } \\
\text { noma } \\
\text { mol- } \\
\text { GPA }\end{array}$} & \multirow[t]{2}{*}{ Age [years] } & $<70$ & 0.5 \\
\hline & & $\geq 70$ & 0 \\
\hline & \multirow[t]{3}{*}{ KPS [\%] } & $100-90$ & 1 \\
\hline & & 80 & 0.5 \\
\hline & & $\leq 70$ & 0 \\
\hline & \multirow[t]{2}{*}{ Extracranial metastases } & No & 1 \\
\hline & & Yes & 0 \\
\hline & \multirow{3}{*}{$\begin{array}{l}\text { Number of brain } \\
\text { metastases }\end{array}$} & 1 & 1 \\
\hline & & $2-4$ & 0.5 \\
\hline & & $\geq 5$ & 0 \\
\hline & \multirow[t]{2}{*}{ BRAF mutation status } & Positive & 0.5 \\
\hline & & Negative/unknown & 0 \\
\hline
\end{tabular}

distant brain progression, last follow-up visit, or death. All statistical calculations were performed using SPSS Statistics Version 25 (IBM, USA). Outcome analyses and determination of prognostic factors were based on Kaplan-Meier estimates with log-rank tests and the Cox regression method.

A $p$ value of $<0.05$ was defined as the threshold for statistical significance within a confidence interval of $95 \%$.

\section{Results}

\section{Outcomes and toxicity}

The median age was 61 years (range 27-80); 36 females $(36 / 83,43.4 \%)$ and $47(47 / 83,56.6 \%)$ male patients were treated with SRS. Median follow-up for all patients was 
5.7 months (IQR: 14.5 months); for patients alive, it was 24.6 months (IQR: 25.1 months). Seven patients (7/83, $8.4 \%$ ) were lost to follow-up.

At the time of analysis, $74.7 \%$ of patients (62/83) were deceased, which resulted in a median OS of 9.7 months (95\%-KI 4.7-14.7). Twenty-two patients $(22 / 173,12.7 \%)$ suffered from local failure. LC at 6-months, 12-, and 24 -months was $89 \%, 86 \%$, and $72 \%$, respectively (the median was not reached). Loco-regional failure occurred in 41 patients $(41 / 83,49.4 \%)$, resulting in a median DBC of 8.2 months (95\%-KI 4.7-11.7). Table 2 shows further outcome data.

Radionecrosis was seen by MRI in $9(9 / 83,10.8 \%)$ patients after SRS classified as CTCAE grade 2 in eight cases as CTCAE grade 1 in one case. The median time to occurrence of radionecrosis was 9.0 months (range 2.4-18.8 months). Leptomeningeal failure did not occur in this cohort. Regarding side effects CTCAE grade $\geq 3$, we saw motor disorders in four cases in the first 6 months, and in three after 6 months. No sensory disorders grade $\geq 3$ were reported. Epilepsy was seen in one patient in the first 6 months and in two patients after six months. Other side effects grade $\geq 3$ were nausea, cognitive disorders, and dizziness in one patient each.

\section{GPA assessment}

We calculated the initial GPA and melanoma molGPA, according to the recently published works by Sperduto et al. [4]. The GPA is calculated by adding the score values for the three parameters for age, KPS, extracranial metastases, and the number of brain metastases; the melanoma molGPA by also adding the value of the BRAF status (Table 3). Each of the four criteria is given a score of $0,0.5$, or 1.0 , with the best prognosis in patients with a score of 4.0.

\section{Prognostic assessment}

Univariate and multivariate analyses (UVA, MVA) are shown in Table 4. For OS, a KPS $\geq 80 \%$, a positive BRAF mutation status (Fig. 1), a small PTV, the absence of extracranial metastases, as well as a GPA and melanoma molGPA $>2$ (Fig. 2) were prognostic factors. In the MVA, we used only the significant values and excluded two parameters: the initial GPA and its values should only be included

Table 4 Prognostic assessment on OS, LC, and LRC

\begin{tabular}{|c|c|c|c|c|c|c|c|c|}
\hline & \multicolumn{4}{|l|}{ OS } & \multirow{2}{*}{\multicolumn{2}{|c|}{$\frac{\mathrm{LC}}{\mathrm{UVA}}$}} & \multirow{2}{*}{\multicolumn{2}{|c|}{$\frac{\mathrm{DBC}}{\mathrm{UVA}}$}} \\
\hline & \multicolumn{2}{|l|}{ UVA } & \multicolumn{2}{|l|}{ MVA } & & & & \\
\hline & $P$-value & $\begin{array}{l}\mathrm{HR} \\
(95 \% \mathrm{CI})\end{array}$ & $P$-value & $\begin{array}{l}\mathrm{HR} \\
(95 \% \mathrm{CI})\end{array}$ & $P$-value & $\begin{array}{l}\mathrm{HR} \\
(95 \% \mathrm{CI})\end{array}$ & $P$-value & $\begin{array}{l}\mathrm{HR} \\
(95 \% \mathrm{CI})\end{array}$ \\
\hline Age at $\mathrm{RT}^{*}$ & 0.707 & $0.99(0.98-1.02)$ & - & & 0.201 & $0.98(0.94-1.01)$ & 0.642 & $0.99(0.97-1.02)$ \\
\hline KPS (<80\% vs. $\geq 80 \%)$ & $0.024 *$ & $2.28(1.11-4.65)$ & - & & 0.299 & $0.04(0.00-16.77)$ & 0.680 & $0.74(0.18-3.09)$ \\
\hline $\begin{array}{l}\text { Time from primary diagnosis to } \\
\text { brain metastasis * }\end{array}$ & 0.186 & $1.00(0.99-1.00)$ & - & & 0.562 & $1.00(0.99-1.00)$ & 0.770 & $1.00(1.00-1.01)$ \\
\hline $\begin{array}{l}\text { BRAF mutation (yes vs. no/ } \\
\text { unknown) }\end{array}$ & $0.004 *$ & $3.04(1.44-6.40)$ & - & & 0.703 & $1.18(0.50-2.80)$ & 0.834 & $0.93(0.49-1.78)$ \\
\hline BRAF mutation (yes vs. no) & 0.050 & $0.44(0.19-1.00)$ & - & & 0.316 & $0.51(0.14-1.90)$ & 0.929 & $0.97(0.46-2.03)$ \\
\hline $\begin{array}{l}\text { simultaneously immune therapy } \\
\text { (yes vs. no) }\end{array}$ & 0.478 & $1.14(0.79-1.66)$ & - & & 0.624 & $1.15(0.67-1.97)$ & 0.862 & $0.97(0.65-1.43)$ \\
\hline Prior WBRT (yes vs. no) & 0.061 & $0.76(0.56-1.01)$ & - & & 0.086 & $0.67(0.43-1.06)$ & 0.982 & $1.00(0.64-1.54)$ \\
\hline PTV* & $<0.001 *$ & $1.12(1.05-1.19)$ & 0.001* & $\begin{array}{l}1.11 \\
(1.04-1.18)\end{array}$ & $0.045^{*}$ & $1.09(1.00-1.19)$ & 0.394 & $1.05(0.94-1.16)$ \\
\hline $\mathrm{D}_{\max } *$ & 0.163 & $0.86(0.70-1.06)$ & - & & $0.027 *$ & $0.67(0.47-0.96)$ & 0.058 & $1.34(0.99-1.81)$ \\
\hline $\begin{array}{l}\text { Extra-cranial metastases (yes } \\
\text { vs. no) }\end{array}$ & $0.004 *$ & $0.57(0.39-0.84)$ & - & & 0.065 & $0.50(0.24-1.04)$ & 0.053 & $0.67(0.44-1.01)$ \\
\hline $\begin{array}{l}\text { Number of all brain mets (1 } \\
\text { vs. }>1)\end{array}$ & 0.538 & $0.83(0.46-1.50)$ & - & & 0.164 & $0.51(0.20-1.32)$ & $0.037^{*}$ & $2.52(1.06-6.01)$ \\
\hline GPA (0-2 vs. $2.5-4)$ & 0.043* & $1.97(1.02-3.81)$ & - & & 0.736 & $0.84(0.31-2.29)$ & $0.044 *$ & $0.43(0.19-0.98)$ \\
\hline $\begin{array}{l}\text { Melanoma molGPA (0-2 vs. } \\
2.5-4)\end{array}$ & 0.001* & $0.34(0.18-0.63)$ & $0.001 *$ & $\begin{array}{l}2.92 \\
(1.54-5.54)\end{array}$ & 0.996 & $1.00(0.43-2.38)$ & 0.409 & $1.32(0.68-2.56)$ \\
\hline
\end{tabular}

KPS Karnofsky performance score, BRAF Serine/threonine protein kinase, encoded on chromosome 7q34, that activates the MAP kinase/ERKsignaling pathway, GPA Graded prognostic assessment according to [27], WBRT Whole brain radiotherapy, PTV Planning target volume, $D_{m a x}$ Maximum dose to the PTV, UVA Univariate analysis, MVA Multivariate analysis, * continues variable 


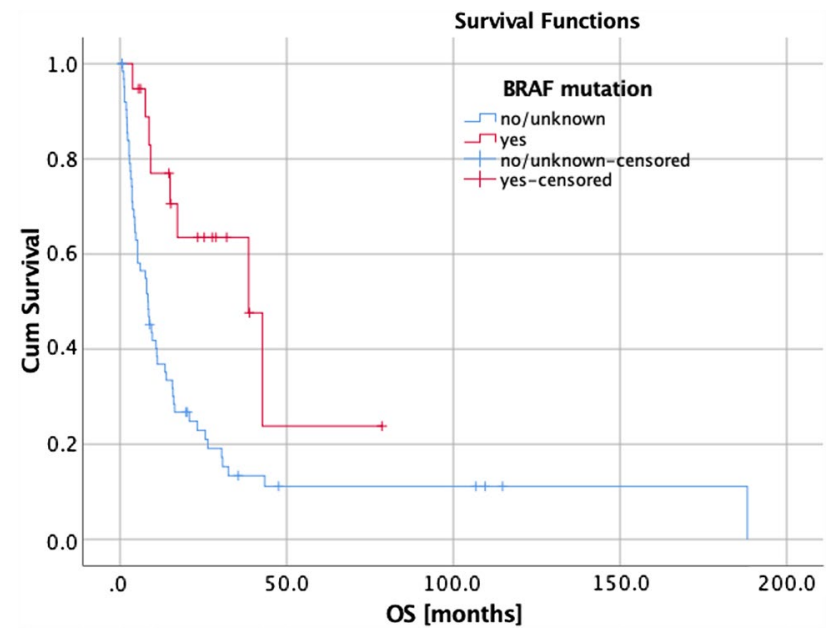

Fig. 1 Survival curves for BRAF mutation status $(p=0.004)$

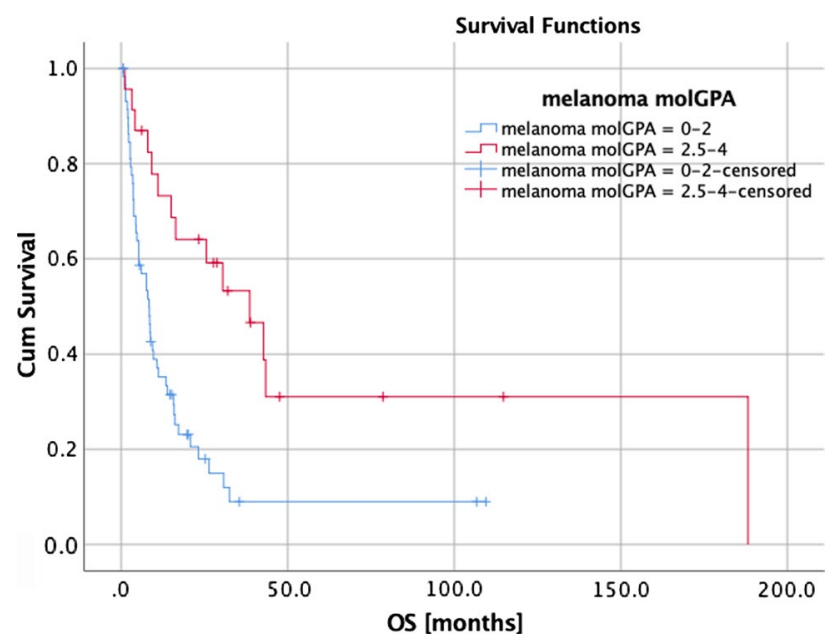

Fig. 2 Survival curves for the melanoma molGPA $(p=0.001)$

once in the MVA, and the extracranial metastases, BRAF status, and KPS as they are part of the molGPA calculation. In the MVA, a small PTV and a melanoma molGPA $>2$ remained significant (Fig. 3).

A small PTV and $D_{\max }$ had a significant influence on LC. The presence of a single brain metastasis, and the initial GPA impacted DBC.

\section{Discussion}

The present study aimed to identify outcomes and prognostic factors of melanoma patients with brain metastasis. Treatment was very well tolerated with low rates of side effects, especially regarding high-grade toxicity. Median OS was 9.7 months, and LC at one year was $86 \%$. The melanoma molGPA showed a significant impact on OS with $p=0.001$

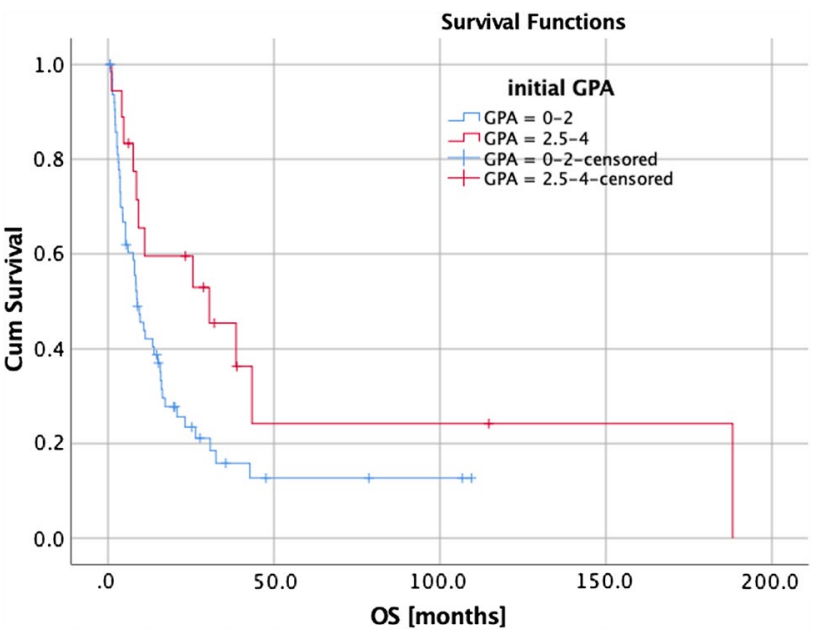

Fig. 3 Survival curves for the initial GPA $(p=0.043)$

with a higher molGPA associated with an improved OS. Further predictors of outcome include a KPS $\geq 80 \%$, a positive BRAF mutation status, a small PTV, the absence of extracranial metastases.

Predictors for OS in patients with malignant melanoma metastatic to the brain have been published previously and include LDH levels, age, KPS, number of brain metastasis, leptomeningeal spread, presence of extracranial metastases, melanoma ulceration, histology and neurologic symptoms [28]. Median OS in our study was 9.7 months, which is equal compared to the study of Yu et al. in 2002 [4, 28] as well as Raizer et al. in 2008 [4] and close to other relevant studies in this field (Table 5). In our study, LC, after one year, was $86 \%$, it should be mentioned that early death may preclude the development of progression. Other groups, Gaudy-Marqueste et al. and Mori et al., achieved comparable excellent control rates of $84 \%$ and $90 \%$ [29-34]. Powell et al. reported an LC rate of $63 \%$ in 50 examined patients [35]. This OS and $\mathrm{LC}$ rate reinforces the statement that SRS has become a well-established treatment modality with high efficacy as it delivers a high dose to the lesion, even if brain metastases of malignant melanoma have traditionally been considered radioresistant lesions when treated with conventional radiotherapeutic modalities [32].

None of the tested factors impacted LRC, including WBRT. However, this offers only limited information for the present cohort as only $21 \%$ of patients were pre-treated with WBRT.

This has to be seen in contrast to other studies of different tumor types which have demonstrated increased distant brain control with WBRT. Presumably this is a dose effect with malignant melanoma and thus shows similar results to the paper published in 2019 by Hong et al. that adjuvant WBRT does not provide clinical benefit in terms of distant intracranial control [36]. 
Table 5 Selection of studies with local control rates after 1 year

\begin{tabular}{|c|c|c|c|c|c|}
\hline Publication, Year & Patients & Primary & Therapy & $\begin{array}{l}\text { Median OS } \\
\text { [months] }\end{array}$ & LC after 1 year \\
\hline Yu et al. [35], 2002 & 122 & MM & GKR & 9.1 & $\begin{array}{l}86 \%(<1 \mathrm{~cm}) \\
63 \%(3-9 \mathrm{~cm})\end{array}$ \\
\hline Gaudy-Marqueste et al. [37], 2006 & 106 & MM & GKR & 4.1 & $69 \%$ \\
\hline Neal et al. [59], 2014 & 129 & MM & GKR & NR & $81 \%$ \\
\hline Rades et al. [60], 2014 & 54 & MM & SRS & NR & $\begin{array}{l}100 \%(21-22.5 \mathrm{~Gy}), \\
72 \%(20 \mathrm{~Gy})\end{array}$ \\
\hline Gieger et al. [61], 1997 & 12 & MM & SRS & NR & $57 \%$ \\
\hline Brown et al. [62], 2008 & 39 & $\mathrm{MM} / \mathrm{RCC}$ & SRS & 14.2 & $100 \%$ (6 months) \\
\hline Mori et al. [38], 1998 & 60 & MM & SRS & 7.0 & $90 \%$ \\
\hline Radbill et al. [63], 2004 & 51 & MM & GKR & 5.7 & $81 \%$ \\
\hline Lavine et al. [64], 1999 & 45 & MM & GKR & 43.0 & $97 \%$ \\
\hline Sia et al. [65], 2015 & 162 & various & SRS & 5.1 & $82 \%$ \\
\hline Likhacheva et al. [66], 2013 & 251 & various & SRS & 11.1 & $95 \%$ \\
\hline Chang et al. [67], 2009 & 58 & various & SRS & 15.2 & $67 \%$ \\
\hline Andrews et al. [42], 2004 & 331 & various & WBRT + SRS Boost & NR & $82 \%$ \\
\hline This work & 83 & MM & SRS & 9.7 & $86 \%$ \\
\hline
\end{tabular}

$M M$ Malignant melanoma, $R C C$ Renal cell cancer; SRS Stereotactic radiosurgery, GKR Gamma knife radiosurgery, NR Not reported

Regarding radionecrosis, it occurred in $10.8 \%$ of our patients after SRS, which is comparable with other studies [37, 38]; all cases of low CTCAE grade.

The results showed that OS was significantly impacted by the BRAF mutation status $(p=0.004)$, with a positive BRAF mutation status associated with an improved OS. However, it offers only limited information for the present cohort as $45 \%$ of patients had an unknown BRAF mutation status.

Bian et al. evaluated in 2016, 401 patients with metastatic melanoma treated with SRS for metastases to the brain. A higher tumor volume was associated with worse survival, as in our study [39]. In multivariate analysis, a small PTV and a melanoma molGPA $>2$ remained significant. Historically, the original GPA has been calculated with the four parameters: age, KPS, number of brain metastases, and extracranial metastases (Table 3). It has been proven useful by Sperduto et al. in a study with 140 patients [21]. This impact could also be shown in our present evaluation $(p=0.043)$. After further research, Sperduto et al. identified five significant prognostic factors for survival (age, KPS, extracranial metastases, number of brain metastases, and BRAF status) for melanoma patients, and rebuilt the initial GPA to the melanoma molGPA [40-45]. They validated the score on a cohort of 823 melanoma patients with newly diagnosed brain metastases in a multi-institutional retrospective analysis. This review demonstrates that this disease-specific assessment, including relevant outcome and prognostic biomarkers, provides a better and reliable prognostic score. This strengthens the trend that incorporating biomarkers, which show influence on OS based on the latest research, will facilitate clinical decision making regarding whether and which treatment is appropriate. It will also be useful for stratification of future clinical trials [46].

Immunomodulators and targeted agents against mutations in the BRAF gene have become established treatment for patients with metastatic melanoma, offering a survival benefit [4]. The substances also appear to have a positive effect on intracerebral control. Dummer et al. 2014 in their small collective $(n=24)$ treated with vemurafenib, $37 \%$ achieved an intracranial tumor decrease of $>30 \%$ [47]. In the review of Ly et al. [28], treatment with BRAF inhibitors was associated with improved local control after SRS in 52 patients with melanoma and brain metastases compared to no treatment of BRAF inhibitors.

Further prognostic factors such as the number and symptoms of cerebral metastases, serum LDH and S100 levels, extracranial metastasis, and KPS receive more and more attention in interdisciplinary therapy planning. The trend is moving more and more in the direction of individualized treatment. Personalized medicine has the potential to tailor therapy with the best response and highest safety margin to ensure better patient care. By enabling each patient to receive earlier diagnoses, risk assessments, and optimal treatments, Personalized medicine holds promise for improving health care while also lowering costs [14]. Current clinical studies are investigating new therapy options for melanoma patients with brain metastases such as PD-1 antibodies, ipilimumab plus nivolumab, BRAF inhibitors plus MEK inhibitors, as well as stereotactic radiotherapy in combination with immunotherapy or targeted therapy [48]. Ipilimumab, pembrolizumab, and nivolumab are approved for the treatment of unresectable or metastatic melanoma, as 
is the combination of nivolumab and ipilimumab, which in the CheckMate 067 trial outperformed the respective monotherapies in terms of response and progression-free survival, even though it was significantly more toxic [49]. Therapy with an antibody directed against PD-1 with or without the addition of ipilimumab is the standard therapy at least for advanced melanoma without BRAF mutation. The combination of BRAF/MEK inhibitors shows the highest response rates of all melanoma therapies. This treatment is particularly suitable as first-line therapy for patients with symptomatic BRAF mutated melanoma with rapid progression and high therapeutic pressure. However, the duration of therapy is usually limited by the development of resistance, which occurs in the majority of patients during treatment. Most of the resistance mechanisms lead to a reactivation of the MAP kinase signaling pathway $[50,51]$.

Chemotherapy plays a subordinate role in the treatment of melanoma brain metastases. Before the approval of ipilimumab, prior to 2011, treatment with dacarbazine was considered the standard of care for patients with inoperable melanoma metastases [52]. Alternatively, temozolomide or fotemustin was used without leading to a significant prolongation of overall survival [53].

Generally, SRS is widely accepted in patients with 1-3, maximal 4 lesions. There is increasing evidence that even in multiple lesions, SRS can be highly effective. Considering the benefit of high local doses for radioresistant melanoma lesions, multiple SRS may be more effective compared to WBRT in those patients. Only recently, Yamamoto et al. [54] reported excellent outcomes of radiosurgery, even in patients with ten or more lesions. Further analyses have shown that outcome in patients with 5-10 lesions is non-inferior to patients with 2-4 lesions [48, 55]. Importantly, the addition of novel drugs such as TK-Is or other immunomodulators can be applied safely in the context of SRS without enhanced toxicity [56]. Here for SRS can be seen as a bridge to systemic treatment and should be considered for palliative therapy for patients with unresectable or metastatic melanoma. After an international meta-analysis of Lehrer et al. in 2018, 17 studies across 15 institutes in 3 countries have been analyzed and shown that concurrent administration of immune checkpoint inhibitors and SRS does not appear to be associated with untoward rates of radionecrosis with a possible survival advantage observed. Additionally, enhanced regional brain control within the brain with excellent rates of 1-year LC may be associated with concurrent therapy [57, 58]. This argues for non-invasive treatment of brain metastases even in advanced and extra-cranially spread disease to prevent progression and subsequent neurological deterioration, while treating the underlying disease effectively.

In conclusion, despite the still poor prognosis for brain metastasis in malignant melanoma, SRS is an effective treatment method. The BRAF biomarker and prognostic scores, such as the melanoma molGPA, could be used as tools in treatment decision making. The present survival outcomes support the use of disease-specific melanoma molGPA as reliable prognostic score. Immunotherapy is continuously revolutionizing outcome in patients with melanoma, making noninvasive and highly effective local treatments for brain metastases more relevant, even if the benefits and risks of the combination of radiotherapy with BRAF-targeted agents are also yet to be established. However, all patients with melanoma brain metastases should be discussed in a multidisciplinary team to make the best recommendations regarding patients' treatment and care. Key research opportunities for future clinical trials are SRS in combination with BRAF pathway inhibitors and systemic therapies.

Funding Open Access funding enabled and organized by Projekt DEAL.

\section{Declarations}

Conflict of interest No conflicts of interest.

Ethical approval The ethics approval from the german ethics committee is under the file reference $257 / 16 \mathrm{~S}$ dated 01.06 .2016 for this project. All data have been collected retrospectively from anonymized data.

Informed consent For this type of study formal consent is not required.

Open Access This article is licensed under a Creative Commons Attribution 4.0 International License, which permits use, sharing, adaptation, distribution and reproduction in any medium or format, as long as you give appropriate credit to the original author(s) and the source, provide a link to the Creative Commons licence, and indicate if changes were made. The images or other third party material in this article are included in the article's Creative Commons licence, unless indicated otherwise in a credit line to the material. If material is not included in the article's Creative Commons licence and your intended use is not permitted by statutory regulation or exceeds the permitted use, you will need to obtain permission directly from the copyright holder. To view a copy of this licence, visit http://creativecommons.org/licenses/by/4.0/.

\section{References}

1. Hayat MA Brain Metastases from Primary Tumors Volume Three: Epidemiology, Biology, and Therapy of Melanoma and Other Cancers, ed. M.A. Hayat. 2016 Academic Press. 384.

2. Linskey ME, et al. The role of stereotactic radiosurgery in the management of patients with newly diagnosed brain metastases: a systematic review and evidence-based clinical practice guideline. J Neurooncol. 2010;96(1):45-68.

3. Spencer K, Hall A, Jain P. Brain metastases. Clin Med (Lond). 2014;14(5):535-7.

4. Sperduto CM, et al. A validation study of a new prognostic index for patients with brain metastases: the graded prognostic assessment. J Neurosurg. 2008;109(Suppl):87-9.

5. Sperduto PW, et al. Estimating survival in patients with lung cancer and brain metastases: an update of the graded prognostic 
assessment for lung cancer using molecular markers (Lung-molGPA). JAMA Oncol. 2017;3(6):827-31.

6. Nieder $\mathrm{C}$, et al. Validation of the graded prognostic assessment for melanoma using molecular markers (Melanoma-molGPA). J Clin Med Res. 2018;10(3):178-81.

7. Sperduto PW, et al. Effect of tumor subtype on survival and the graded prognostic assessment for patients with breast cancer and brain metastases. Int J Radiat Oncol Biol Phys. 2012;82(5):2111-7.

8. O'Beirn $\mathrm{M}$ et al. The expanding role of radiosurgery for brain metastases. Medicines (Basel), 2018.

9. Kalkanis SN, Linskey ME. Evidence-based clinical practice parameter guidelines for the treatment of patients with metastatic brain tumors: introduction. J Neurooncol. 2010;96(1):7-10.

10. Badiyan SN, Regine WF, Mehta M. Stereotactic radiosurgery for treatment of brain metastases. J Oncol Pract. 2016;12(8):703-12.

11. Kocher M, et al. Stereotactic radiosurgery for treatment of brain metastases. A report of the DEGRO working group on stereotactic radiotherapy. Strahlenther Onkol. 2014;190(6):521-32.

12. Schvartsman $G$, et al. Incidence, patterns of progression, and outcomes of preexisting and newly discovered brain metastases during treatment with anti-PD-1 in patients with metastatic melanoma. Cancer. 2019;125(23):4193-202.

13. Fonkem E, et al. Melanoma brain metastasis: overview of current management and emerging targeted therapies. Expert Rev Neurother. 2012;12(10):1207-15.

14. Corrie, et al. Management of melanoma. Br Med Bull. 2014;111(1):149-62.

15. Long GV, et al. Dabrafenib in patients with Val600Glu or Val600Lys BRAF-mutant melanoma metastatic to the brain (BREAK-MB): a multicentre, open-label, phase 2 trial. Lancet Oncol. 2012;13(11):1087-95.

16. Davies MA, et al. Dabrafenib plus trametinib in patients with BRAF(V600)-mutant melanoma brain metastases (COMBI$\mathrm{MB})$ : a multicentre, multicohort, open-label, phase 2 trial. Lancet Oncol. 2017;18(7):863-73.

17. Long GV, et al. Combination nivolumab and ipilimumab or nivolumab alone in melanoma brain metastases: a multicentre randomised phase 2 study. Lancet Oncol. 2018;19(5):672-81.

18. Tawbi HA, et al. Combined nivolumab and ipilimumab in melanoma metastatic to the brain. N Engl J Med. 2018;379(8):722-30.

19. Davies $\mathrm{H}$, et al. Mutations of the BRAF gene in human cancer. Nature. 2002;417(6892):949-54.

20. Seoane J, De Mattos-Arruda L. Brain metastasis: new opportunities to tackle therapeutic resistance. Mol Oncol. 2014;8(6):1120-31.

21. Lwu S, et al. Stereotactic radiosurgery for the treatment of melanoma and renal cell carcinoma brain metastases. Oncol Rep. 2013;29(2):407-12.

22. Ajithkumar T, et al. Evolving treatment options for melanoma brain metastases. Lancet Oncol. 2015;16(13):e486-97.

23. Schultz ES, Schuler G. Malignant melanoma. Diagnosis and therapy. HNO. 2005;53(11):928-39.

24. Eaton DJ, et al. Stereotactic radiosurgery for benign brain tumors: results of multicenter benchmark planning studies. Pract Radiat Oncol. 2018;8(5):e295-304.

25. Han EY et al. Dosimetric comparison of fractionated radiosurgery plans using frameless Gamma Knife ICON and CyberKnife systems with linear accelerator-based radiosurgery plans for multiple large brain metastases. J Neurosurg 2019: p. 1-7.

26. Yomo $\mathrm{S}$ et al. The impact of EGFR-TKI use on clinical outcomes of lung adenocarcinoma patients with brain metastases after Gamma Knife radiosurgery: a propensity score-matched analysis based on extended JLGK0901 dataset (JLGK0901-EGFR-TKI). J Neurooncol, 2019.
27. Lehrer EJ, et al. Treatment of brain metastases with stereotactic radiosurgery and immune checkpoint inhibitors: an international meta-analysis of individual patient data. Radiother Oncol. 2019;130:104-12.

28. Sperduto PW, et al. Estimating survival in melanoma patients with brain metastases: an update of the graded prognostic assessment for melanoma using molecular markers (melanoma-molGPA). Int J Radiat Oncol Biol Phys. 2017;99(4):812-6.

29. Fife KM, et al. Determinants of outcome in melanoma patients with cerebral metastases. J Clin Oncol. 2004;22(7):1293-300.

30. Zakrzewski J, et al. Clinical variables and primary tumor characteristics predictive of the development of melanoma brain metastases and post-brain metastases survival. Cancer. 2011;117(8):1711-20.

31. Eigentler TK, et al. Number of metastases, serum lactate dehydrogenase level, and type of treatment are prognostic factors in patients with brain metastases of malignant melanoma. Cancer. 2011;117(8):1697-703.

32. Raizer JJ, et al. Brain and leptomeningeal metastases from cutaneous melanoma: survival outcomes based on clinical features. Neuro Oncol. 2008;10(2):199-207.

33. Staudt M, et al. Determinants of survival in patients with brain metastases from cutaneous melanoma. Br J Cancer. 2010;102(8):1213-8.

34. Davies MA, et al. Prognostic factors for survival in melanoma patients with brain metastases. Cancer. 2011;117(8):1687-96.

35. $\mathrm{Yu} \mathrm{C}$, et al. Metastatic melanoma to the brain: prognostic factors after gamma knife radiosurgery. Int J Radiat Oncol Biol Phys. 2002;52(5):1277-87.

36. Hong AM, et al. Adjuvant whole-brain radiation therapy compared with observation after local treatment of melanoma brain metastases: a multicenter, randomized phase III Trial. J Clin Oncol. 2019;37(33):3132-41.

37. Gaudy-Marqueste $\mathrm{C}$, et al. Gamma-Knife radiosurgery in the management of melanoma patients with brain metastases: a series of 106 patients without whole-brain radiotherapy. Int J Radiat Oncol Biol Phys. 2006;65(3):809-16.

38. Mori Y, et al. Stereotactic radiosurgery for cerebral metastatic melanoma: factors affecting local disease control and survival. Int J Radiat Oncol Biol Phys. 1998;42(3):581-9.

39. Powell JW, et al. Gamma Knife surgery in the management of radioresistant brain metastases in high-risk patients with melanoma, renal cell carcinoma, and sarcoma. J Neurosurg. 2008;109(Suppl):122-8.

40. Sanghavi SN, et al. Radiosurgery for patients with brain metastases: a multi-institutional analysis, stratified by the RTOG recursive partitioning analysis method. Int J Radiat Oncol Biol Phys. 2001;51(2):426-34.

41. Sneed, P.K., et al., A multi-institutional review of radiosurgery alone vs. radiosurgery with whole brain radiotherapy as the initial management of brain metastases. Int J Radiat Oncol Biol Phys, 2002. 53(3): p. 519-26.

42. Andrews DW, et al. Whole brain radiation therapy with or without stereotactic radiosurgery boost for patients with one to three brain metastases: phase III results of the RTOG 9508 randomised trial. Lancet. 2004;363(9422):1665-72.

43. Manon R, et al. Phase II trial of radiosurgery for one to three newly diagnosed brain metastases from renal cell carcinoma, melanoma, and sarcoma: an Eastern cooperative oncology group study (E 6397). J Clin Oncol. 2005;23(34):8870-6.

44. Kocher M, et al. Adjuvant whole-brain radiotherapy versus observation after radiosurgery or surgical resection of one to three cerebral metastases: results of the EORTC 22952-26001 study. J Clin Oncol. 2011;29(2):134-41. 
45. Frazier JL, et al. Stereotactic radiosurgery in the management of brain metastases: an institutional retrospective analysis of survival. Int J Radiat Oncol Biol Phys. 2010;76(5):1486-92.

46. Bian SX, et al. Prognostic factors for melanoma brain metastases treated with stereotactic radiosurgery. J Neurosurg. 2016;125(Suppl 1):31-9.

47. Sperduto PW, et al. Diagnosis-specific prognostic factors, indexes, and treatment outcomes for patients with newly diagnosed brain metastases: a multi-institutional analysis of 4,259 patients. Int J Radiat Oncol Biol Phys. 2010;77(3):655-61.

48. Dummer R, et al. Vemurafenib in patients with BRAF(V600) mutation-positive melanoma with symptomatic brain metastases: final results of an open-label pilot study. Eur J Cancer. 2014;50(3):611-21.

49. Larkin J, et al. Five-year survival with combined nivolumab and ipilimumab in advanced melanoma. N Engl J Med. 2019;381(16):1535-46.

50. Larkin J, et al. Combined nivolumab and ipilimumab or monotherapy in untreated melanoma. N Engl J Med. 2015;373(1):23-34.

51. Ascierto PA, et al. Cobimetinib combined with vemurafenib in advanced BRAF(V600)-mutant melanoma (coBRIM): updated efficacy results from a randomised, double-blind, phase 3 trial. Lancet Oncol. 2016;17(9):1248-60.

52. Ly D, et al. Local control after stereotactic radiosurgery for brain metastases in patients with melanoma with and without BRAF mutation and treatment. J Neurosurg. 2015;123(2):395-401.

53. Vogenberg FR, Isaacson Barash C, Pursel M Personalized medicine: part 1: evolution and development into theranostics. P T, 2010. 35(10): 560-76.

54. Ascierto PA, et al. Phase II trial (BREAK-2) of the BRAF inhibitor dabrafenib (GSK2118436) in patients with metastatic melanoma. J Clin Oncol. 2013;31(26):3205-11.

55. McArthur GA, et al. Safety and efficacy of vemurafenib in BRAF(V600E) and BRAF(V600K) mutation-positive melanoma (BRIM-3): extended follow-up of a phase 3, randomised, openlabel study. Lancet Oncol. 2014;15(3):323-32.

56. Yamamoto M, et al. Stereotactic radiosurgery for patients with multiple brain metastases: a case-matched study comparing treatment results for patients with 2-9 versus 10 or more tumors. J Neurosurg. 2014;121(Suppl):16-25.

57. Serizawa $\mathrm{T}$ et al. Local tumor progression treated with Gamma Knife radiosurgery: differences between patients with 2-4 versus
5-10 brain metastases based on an update of a multi-institutional prospective observational study (JLGK0901). J Neurosurg, 2019: $1-10$.

58. Cao H et al. Dosimetric comparisons of different hypofractionated stereotactic radiotherapy techniques in treating intracranial tumors $>3 \mathrm{~cm}$ in longest diameter. J Neurosurg, 2019: 1-9.

59. Neal MT, et al. Predictors of survival, neurologic death, local failure, and distant failure after gamma knife radiosurgery for melanoma brain metastases. World Neurosurg. 2014;82(6):1250-5.

60. Rades D, et al. Radiosurgery alone for 1-3 newly-diagnosed brain metastases from melanoma: impact of dose on treatment outcomes. Anticancer Res. 2014;34(9):5079-82.

61. Gieger M, et al. Response of intracranial melanoma metastases to stereotactic radiosurgery. Radiat Oncol Investig. 1997;5(2):72-80.

62. Brown PD, et al. Stereotactic radiosurgery for patients with "radioresistant" brain metastases. Neurosurgery. 2008;62(Suppl 2):790-801.

63. Radbill AE, et al. Initial treatment of melanoma brain metastases using gamma knife radiosurgery: an evaluation of efficacy and toxicity. Cancer. 2004;101(4):825-33.

64. Lavine SD et al. Gamma knife radiosurgery for metastatic melanoma: an analysis of survival, outcome, and complications. Neurosurgery, 1999. 44(1): 59-64; discussion 64-6.

65. Sia J, et al. Stereotactic radiosurgery for 318 brain metastases in a single Australian centre: the impact of histology and other factors. J Clin Neurosci. 2015;22(2):303-7.

66. Likhacheva A, et al. Predictors of survival in contemporary practice after initial radiosurgery for brain metastases. Int J Radiat Oncol Biol Phys. 2013;85(3):656-61.

67. Chang EL, et al. Neurocognition in patients with brain metastases treated with radiosurgery or radiosurgery plus wholebrain irradiation: a randomised controlled trial. Lancet Oncol. 2009;10(11):1037-44.

Publisher's Note Springer Nature remains neutral with regard to jurisdictional claims in published maps and institutional affiliations. 